\title{
Burden of non-alcoholic steatohepatitis among children with type 2 diabetes mellitus
}

\author{
Lauren Parlett ${ }^{1}$, Qinli Ma ${ }^{*}$, Qian Shi ${ }^{1}$, Geoffrey Crawford ${ }^{2}$, Laura Herrera Scott ${ }^{2}$, Vincent Willey ${ }^{1}$ and Abiy Agiro ${ }^{1}$
}

\begin{abstract}
This claims-based retrospective cohort study examined the prevalence and incremental impact of non-alcoholic steatohepatitis among children with type 2 diabetes mellitus in the United States. Although diagnoses of non-alcoholic steatohepatitis were not common among diabetic children, it was associated with significantly higher incremental healthcare cost and risk of hospitalization.
\end{abstract}

Keywords: Non-alcoholic steatohepatitis, Type 2 diabetes mellitus, Children, Healthcare utilization and cost

\section{Introduction}

Non-alcoholic steatohepatitis (NASH) and type 2 diabetes mellitus (T2DM) share insulin resistance and hyperinsulinemia from a physiologic perspective. Over the past two decades, clinicians have reported an increasing prevalence of comorbid childhood NASH and T2DM [1]. Prevalence of T2DM is estimated to be $11 \%$ among children with a definite NASH diagnoses (biopsy-verified) and 7\% among children with borderline or definite NASH [1]. However, prevalence and incremental impact of NASH among children with T2DM are less known.

\section{Methods}

This retrospective cohort study characterized members under the age of 18 with NASH and T2DM (with or without NASH) using a large, geographically diverse, longitudinal database of medical and pharmacy claims from commercial plans in 14 states and Medicaid claims in 22 states in the United States. We examined all children with a claims-based diagnosis of NASH and/or T2DM between October 1, 2015 and October 31, 2017 who had at least 12 months of continuous health plan enrollment prior to diagnosis.

\footnotetext{
${ }^{*}$ Correspondence: qma@healthcore.com

${ }^{1}$ HealthCore, Inc, Wilmington, DE, USA

Full list of author information is available at the end of the article
}

Follow-up occurred until the end of the study period (October 31, 2018), end of insurance enrollment, death, or the occurrence of a hepatic-related outcome (cirrhosis, hepatocellular carcinoma, liver transplant). Follow-up univariate and multivariable analyses assessing cost and healthcare utilization were performed on a greedy nearest neighbor propensity score-matched 1:1 study sample consisting of NASH+T2DM and T2DM only groups. Variables used for propensity score matching are listed in Footnote A in Table 2. The study did not require institutional review board review as it was not considered human subject research and only de-identified data were used.

\section{Results}

Data in Table 1 show different co-occurrence estimates, depending on the base population. Almost 10\% (50/519) of children with NASH had T2DM and 0.6\% (50/8124) of children with T2DM had NASH. Metabolic syndrome baseline comorbidities revealed that children diagnosed with NASH + T2DM presented similar to children with NASH only (higher prevalence of obesity and dyslipidemia) as compared to children with T2DM only (with the exception of systemic hypertension). Compared to T2DM only, members with NASH + T2DM have more than three times the odds of systemic hypertension (Odds Ratio $=3.51 ; 95 \%$ confidence interval (CI) 1.88, 6.53). The hepatic outcomes (e.g., cirrhosis) occurred 
Table 1 Demographic and clinical characteristics of children (commercial and Medicaid) with NASH, T2DM or both during 12 month baseline period (before matching)

\begin{tabular}{|c|c|c|c|c|c|}
\hline & NASH only $y^{a, b}$ & $\mathrm{NASH}+\mathrm{T} 2 \mathrm{DM}^{\mathrm{a}, \mathrm{b}}$ & T2DM only ${ }^{a}$ & $\begin{array}{l}\text { Odds Ratio }(95 \% \mathrm{Cl}) \\
\mathrm{NASH}+\mathrm{T} 2 \mathrm{DM} \text { compared } \\
\text { to NASH only }{ }^{\mathrm{c}}\end{array}$ & $\begin{array}{l}\text { Odds Ratio }(95 \% \mathrm{Cl}) \\
\mathrm{NASH}+\mathrm{T} 2 \mathrm{DM} \\
\text { compared to T2DM } \\
\text { only }^{\mathrm{C}}\end{array}$ \\
\hline Sample size, N & 469 & 50 & 8074 & & \\
\hline Female, n (\%) & $166(35)$ & $29(58)$ & $4355(54)$ & $2.52(1.39-4.56)$ & $1.18(0.67-2.07)$ \\
\hline Age, mean (SD) & $12.4(3.2)$ & $14.5(2.5)$ & $12.6(4.0)$ & - & - \\
\hline Age, median (IQR) & $13(5)$ & $15(2)$ & $14(5)$ & - & - \\
\hline \multicolumn{6}{|l|}{ Age category, n (\%) } \\
\hline $0-2$ & $\leq 10$ & $\leq 10$ & $82(1)$ & - & - \\
\hline $3-4$ & $\leq 10$ & $\leq 10$ & $369(5)$ & - & - \\
\hline $5-11$ & $177(38)$ & $\leq 10$ & $2069(26)$ & - & - \\
\hline $12-17$ & $286(61)$ & $44(88)$ & $5554(69)$ & $4.69(1.96-11.23)$ & $3.33(1.42-7.82)$ \\
\hline \multicolumn{6}{|l|}{ Health Plan, n (\%) } \\
\hline Medicaid $(N=4514)$ & $353(75)$ & $36(72)$ & $4125(51)$ & $0.85(0.44-1.62)$ & $2.46(1.33-4.57)$ \\
\hline Commercial $(N=4079)$ & $116(25)$ & $14(28)$ & $3949(49)$ & $1.18(0.62-2.27)$ & $0.41(0.22-0.75)$ \\
\hline \multicolumn{6}{|c|}{ Comorbidities at baseline, n (\%) } \\
\hline Obesity & $308(66)$ & $37(74)$ & $2260(28)$ & $1.49(0.77-2.88)$ & $7.32(3.88-13.80)$ \\
\hline Dyslipidemia & $128(27)$ & $15(30)$ & $1022(13)$ & $1.14(0.60-2.16)$ & $2.96(1.61-5.43)$ \\
\hline Systemic hypertension & $28(6)$ & $14(28)$ & $806(10)$ & $6.13(2.96-12.66)$ & $3.51(1.88-6.53)$ \\
\hline
\end{tabular}

$S D$ standard deviation, IQR interquartile range, $\mathrm{Cl}$ confidence interval, NASH non-alcoholic steatohepatitis, T2DM type 2 diabetes mellitus

a NASH was defined as a child having $\geq 1$ claims ICD-10 diagnosis code in the inpatient or emergency department setting or $\geq 2$ claims diagnosis codes in the outpatient setting. T2DM was defined as a child having $\geq 1$ claims ICD-10 diagnosis code in the inpatient or emergency department setting or $\geq 2$ claims diagnosis codes in the outpatient setting or evidence of dispensed antidiabetic medication (except metformin and insulin alone) or metformin fill with presence of T2DM diagnosis code or insulin fill only with presence of T2DM or DM NOS

b Cells containing ten or fewer children are masked due to privacy rules

c Bolded values are statistically significant at $p<.05$

Table 2 Cost and healthcare utilization for childhood T2DM (Commercial and Medicaid) with and without NASH during follow-up period (after matching)

\begin{tabular}{|c|c|c|c|c|}
\hline Measures & $\mathrm{NASH}+\mathrm{T} 2 \mathrm{DM}$ & T2DM only & $\begin{array}{l}\text { Adjusted difference/Incidence rate } \\
\text { ratio (IRR), }(95 \% \mathrm{CI})^{\mathrm{a}}\end{array}$ & P-Value ${ }^{a}$ \\
\hline Sample size, N & 49 & 49 & & \\
\hline Follow-up time, months, median (IQR) & $19(15)$ & $21(16)$ & & \\
\hline All-cause paid cost, PPPM, mean (SD) & $\$ 2,211(\$ 472)$ & $\$ 663(\$ 142)$ & $\$ 1,549(\$ 561, \$ 3,335)$ & $<.0001$ \\
\hline All-cause paid cost, PPPM, median (IQR) & $\$ 576(\$ 1079)$ & $\$ 117(\$ 346)$ & & \\
\hline $\begin{array}{l}\text { All-cause hospitalization, per 1,000 member- } \\
\text { month, IR ( } 95 \% \mathrm{Cl})\end{array}$ & $39.4(28.3,54.9)$ & $16.5(10.1,26.9)$ & $2.4(1.3,4.3)$ & 0.0038 \\
\hline
\end{tabular}

Propensity score was calculated based on 1-year baseline values for age, Deyo-Charlson comorbidity index (DCI), adapted diabetes complications score, outpatient visits, office visits, ER visits, number of prescription classes dispensed, total medical cost, outpatient cost, inpatient cost, ER cost, gender, age group, DCl group, residence region, heart failure, obesity, hypertension, dyslipidemia, polycystic ovarian syndrome, hypothyroidism, sleep apnea, hypopituitarism, and hypogonadism $P P P M$ per patient per month which is a measure of actual paid cost that accounts for varying follow-up time for each child, $I R$ incident ratio, IRR incident rate ratio, $S D$ standard deviation, IQR Interquartile range, Cl confidence interval, NASH non-alcoholic steatohepatitis, T2DM type 2 diabetes mellitus

a Bolded values are statistically significant at $p<.05$

rarely, mainly observed on the T2DM only group, and totaled fewer than our masking threshold of 10.

As shown in Table 2, children with NASH + T2DM had higher all-cause paid costs $(\$ 2,211$ v. $\$ 663$ per patient per month, $\mathrm{p}$ value $<0.0001)$ and more frequent all-cause hospitalizations (Incidence rate ratio $=2.4$; $95 \%$ CI $1.3,4.3$, p value $=0.0038)$ compared to children with T2DM alone. 


\section{Discussion}

In our study population, NASH was associated with significantly higher incremental cost and hospitalization among children with T2DM. However, diagnoses of NASH among children with T2DM appear very rarely. This differs from adults, where NASH is estimated to be present in up to $97 \%$ [2], although the point estimate is wide and varies depending on the population and measurement method. Given histological differences in prepubescent pediatric NASH liver biopsies compared to adults [3] and possible differences in genetic contributions in terms of susceptibility for progression to NASH [4], it is possible that NASH among children with T2DM represents variation of the underlying pathophysiological interplay between insulin resistance/hyperinsulinemia and liver disease compared to what is seen in adults. Alternately, our ascertainment of T2DM and NASH were claims-based, and data was not biopsy-verified NASH; furthermore, follow-up was limited to less than two years, on average. As such, there is possibility of misclassification, especially in the youngest children where T2DM is less prevalent.As recently recommended by the Liver Forum Pediatrics Working Group, emerging therapies for NASH should consider the needs for children with NASH as a distinct group from adults, including comorbid NASH and T2DM, which may constitute a special subgroup [5]. Our data provide meaningful realworld insights for clinicians, health systems, and payers regarding the incremental economic burden of NASH in children. As emerging therapies for NASH appear on the horizon, such analyses are instrumental in appropriately determining their overall value.

\section{Abbreviations \\ Cl: Confidence interval; NASH: Non-alcoholic steatohepatitis; T2DM: Type 2 diabetes mellitus.}

\section{Acknowledgements}

Dianna Hayden and Nianya Liu of HealthCore, Inc. provided data support. Elizabeth Marks provided editorial services. None were compensated beyond their salaries.

\section{Authors' contributions \\ All the authors contributed to the conceptualization and design of this study. $L P, Q M, Q S, A A$, and $V W$ were engaged in data acquisition. $L P, Q M$, and $Q S$ were involved in the statistical analysis. All the authors participated in the data interpretation and revisions of the article. All authors read and approved the final manuscript.}

\section{Funding}

Funding was provided by Anthem (including Amerigroup) and conducted for health plan operations by HealthCore, a health services research subsidiary of Anthem.

\section{Availability of data and materials}

Data from Medicaid and commercially insured members were combined for analysis. Only aggregate data was reported and no individual Medicaid states were identified or reported on. Medicaid state agencies that require approval of this publication have done so.

\section{Declarations}

Ethics approval and consent to participate

This study was conducted in full compliance of relevant provisions of the Health Insurance Portability and Accountability Act. Researchers had access to only a limited dataset that contained no patient identifiers and no patients were contacted directly. The study was exempt from Investigational Review Board oversight under the provisions of Privacy Rule 45 CFR 164.514(e).

\section{Consent for publication}

Not applicable.

\section{Competing interests}

LP, QM and VW are employees of HealthCore, Inc., a wholly owned subsidiary of Anthem, Inc. QS and AA were an employee of HealthCore, Inc. at the time of the study. GC, and LHS are employees of Anthem, Inc. None were compensated for their contribution to this work beyond their salaries. The funding source (Anthem including Amerigroup) had no role in the preparation, approval, or decision to submit the manuscript for publication.

\section{Author details}

${ }^{1}$ HealthCore, Inc, Wilmington, DE, USA. ${ }^{2}$ Anthem, Inc, Indianapolis, IN, USA.

Received: 18 December 2020 Accepted: 13 April 2021

Published online: 21 April 2021

\section{References}

1. Newton KP, Hou J, Crimmins NA, Lavine JE, Barlow SE, Xanthakos SA, Africa J, Behling C, Donithan M, Clark JM, Schwimmer JB, Nonalcoholic Steatohepatitis Clinical Research N. Prevalence of prediabetes and type 2 diabetes in children with nonalcoholic fatty liver disease. JAMA Pediatr. 2016;170(10):e161971. https://doi.org/10.1001/jamapediatrics.2016.1971.

2. Masarone M, Rosato V, Aglitti A, Bucci T, Caruso R, Salvatore T, Sasso FC, Tripodi MF, Persico M. Liver biopsy in type 2 diabetes mellitus: Steatohepatitis represents the sole feature of liver damage. PLOS ONE. 2017;12(6):e0178473. https://doi.org/10.1371/journal.pone.0178473.

3. Kleiner DE, Makhlouf HR. Histology of nonalcoholic fatty liver disease and nonalcoholic steatohepatitis in adults and children. Clin Liver Dis. 2016;20(2):293-312. https://doi.org/10.1016/j.cld.2015.10.011.

4. Dongiovanni P, Anstee QM, Valenti L. Genetic predisposition in NAFLD and NASH: impact on severity of liver disease and response to treatment. Curr Pharm Des. 2013;19(29):5219-38. https://doi.org/10.2174/13816 128113199990381

5. Vos MB, Dimick-Santos L, Mehta R, Omokaro SO, Taminiau J, Schabel E, Kleiner DE, Szitanyi P, Socha P, Schwimmer JB. Factors to consider in development of drugs for pediatric nonalcoholic fatty liver disease. Gastroenterology. 2019. https://doi.org/10.1053/j.gastro.2019.08.048.

\section{Publisher's Note}

Springer Nature remains neutral with regard to jurisdictional claims in published maps and institutional affiliations. 